\title{
POUR UNE TYPOLOGIE DU ROMAN A BYZANCE. LES HEROS ROMANESQUES ET LEUR APPARTENANCE GENERIQUE ${ }^{1}$
}

La notion de genre littéraire, et ce que ce terme a pu signifier pour les Byzantins eux-mêmes, mais aussi, et peut-être surtout, pour nous, chercheurs d'aujourd'hui, constitue un des problèmes majeurs de toute étude sur la littérature byzantine.

Le genre est une notion fort débattue, particulièrement depuis les années 1990, quand des chercheurs, en s'opposant à la tradition littéraire qui n'y voyait qu'un moyen pour classer les productions écrites selon certaines caractéristiques, ont développé des travaux sur les changements des pratiques discursives tout en faisant une place importante aux phénomènes génériques comme des institutions de parole articulant une identité énonciative avec un lieu social ou avec une communauté de locuteurs ${ }^{2}$. Cette approche interprétative met, à la suite des travaux de $\mathrm{H}$. R. Jauss ${ }^{3}$, le récepteur au centre de la démarche et se distingue de la tradition littéraire et rhétorique des genres qui cernait les particularités de textes modèles pour perpétuer l'enseignement de formes canoniques considérées comme admirables.

Pourtant, quand nous parlons de la littérature byzantine, nous avons l'idée qu'elle s'incline servilement devant l'hégémonie du genre et nous partons avec l'idée préconçue que tout produit littéraire de Byzance s'inscrit uniquement et strictement dans les limites génériques déjà codifiées dès l'Antiquité classique ou hellénistique.

Néanmoins, la réalité de la production littéraire byzantine semble défier cette idée ${ }^{4}$ et opère volontairement des glissements ou même des mixtions

${ }^{1}$ Cet article est issu de la communication Mythe et mythe Littéraire à Byzance: Une étude sur Digénis Akritas, présentée au xx ${ }^{\mathrm{e}}$ Congrès International des Etudes Byzantines, Paris, 19-25 août 2001.

2 D. Maingueneau, Le contexte de l'œuvre littéraire. Enonciation, écrivain, société. Paris 1993, ch. 3; J.-C. BEACCo, Les genres textuels dans l'analyse du discours: écriture légitime et communautés translangagières. Langage 105 (1992) 8-27, p.11.

${ }^{3}$ H.-R. Jauss, Pour une esthétique de la réception. Paris 1978.

${ }^{4}$ A. Kazhdan - S. Franklin, Studies on Byzantine Literature of the Eleventh and Twelfth Centuries. Cambridge-Paris 1984. 
génériques $^{5}$ qui parfois peuvent aboutir soit à la naissance de nouveaux genres comme l'hagiographie ou la chronographie, soit à des formes impures du point de vue du genre.

Parmi les textes qui ne trouvent pas facilement leur place dans les taxinomies déjà existantes de la littérature byzantine, nous pouvons en trouver un grand nombre qui, bien qu'ils racontent l'histoire d'un ou de deux héros, ne constituent nullement des textes historiographiques ou des textes épiques. Parallèlement, en raison de leur ancrage dans une réalité historique, leur classification parmi les textes romanesques devient difficile. En ce sens, le texte de Digénis Akritas en constitue un exemple significatif.

Afin de mettre en relief tous les enjeux que comporte une étude sur l'aspect littéraire de Digénis Akritas ${ }^{6}$, il suffit d'examiner deux questions concernant l'étude de ses manuscrits et de son texte, qui ont préoccupé la quasi-majorité des chercheurs et ont monopolisé l'intérêt de la bibliographie, et cela au détriment du texte lui-même et, par conséquent, de son analyse: constitue-t-il un roman, une épopée ou un roman épique? Dans quelle mesure a-t-il imité les romans de la deuxième sophistique, le récit épique, les écrits hagiographiques ou même le récit d'Alexandre?

Afin de pouvoir répondre à la première question, il faudrait s'interroger d'abord sur le terme même de roman et se demander s'il est adéquat de parler de «roman hellénistique», «roman byzantin» ou même «roman occidental» comme si les textes que nous faisions entrer dans ces catégories étaient tellement similaires entre eux que leurs seules différences se limiteraient à l'adjectif - épithète qui en précise la catégorie. Il faudrait d'abord s'enquérir de la réalité générique que recouvre ce terme pour ces genres et, par conséquent, dans une perspective de devenir du genre, examiner de quel romanesque il s'agit vraiment.

La deuxième question se rapporte à la présence ou non des emprunts à d'autres textes ou genres littéraires dans les versions de Digénis Akritas. Cette question, liée à la traditionnelle critique des sources, essaie de dépister, de reconnaître et de démontrer les passages empruntés à d'autres textes qui y sont introduits ou même qui sont juste imités par l'auteur, le copiste ou l'adaptateur dans le but d'anoblir son texte tout en l'inscrivant ainsi dans un continuum littéraire et culturel.

${ }^{5}$ M. Mullett, The Madness of Genre. DOP 46 (1992) 233-243.

${ }^{6}$ Pour les diverses questions qui concernent Digénis Akritas, voir en dernière instance: Digenes Akrites, New Approaches to Byzantine Heroic Poetry (ed. R. Beaton et D. Ricks). London 1993, qui constitue une mise à jour de l'état actuel de la recherche et de la bibliographie. Voir aussi l'introduction de P. Odorico, L'Akrite. L'Epopée byzantine de Digénis Akritas. Versions grecque et slave. Toulouse 2002. 
Certes, les études philologiques constituent toujours une approche précieuse et la seule compétente pour pouvoir restituer un texte véhiculé par un manuscrit, le rendre compréhensible et fiable pour les chercheurs, lui redonner un sens dont il se voit parfois démuni en raison des fautes de l'auteur et/ou du copiste ou même des lacunes qu'il peut contenir. De surcroît, elle est la seule à nous permettre de reconnaître et peut-être aussi d'identifier les passages imités ou copiés à savoir les textes-sources pour le texte en question. Il suffirait de rappeler ici le remarquable travail de S. Alexiou $^{7}$ concernant la version d'Escurial de Digénis Akritas, travail qui a donné un nouvel élan aux études dites «acritiques» en procurant aux chercheurs non seulement un nouveau texte d'ores et déjà cohérent et compréhensible, mais aussi et surtout un nouveau point de vue pour leurs recherches.

Le danger pourtant de cette approche réside dans le fait que le texte peut être appréhendé parfois comme le résultat d'une fusion de passages volés et d'autres imités servilement et sans inspiration ${ }^{8}$. Ainsi, le texte morcelé se voit-il dépossédé de sa double dimension de la réception littéraire, voire de l'accueil de la littérature par l'écriture (auteur) d'une part et par la lecture (lecteur) de l'autre.

Dans cette perspective, nous avons pu constater que le récit de Digénis Akritas (ou devrais-je dire les récits?) est influencé non seulement par le roman ou par l'épopée mais aussi par l'hagiographie. Néanmoins, les ressemblances/influences s'arrêtent là, étant donné que le récit de Digénis Akritas diffère sensiblement d'un récit typiquement romanesque ou épique,

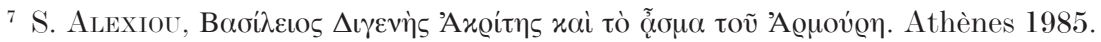

${ }^{8}$ Il ne s'agit pas de minimiser la valeur indéniable de l'approche philologique, mais de souligner le besoin d'élargir l'optique de la traditionnelle critique des sources qui envisageait les mêmes phénomènes d'un point de vue strictement «biographique»: Quels volumes contenait la bibliothèque d'un écrivain? Quels livres avait-il lus? Dans quelle filiation s'inscrivait-il? Les problèmes d'influences, de transmission, d'«hérédité» ou d'héritage réglant cette approche, il s'agit, alors, de réintroduire dans l'optique de la recherche le texte lui-même et en conséquent d'étudier aussi la transformation des sources qui s'opère au sein même du texte au lieu de partir des sources, extérieures à lui pour ensuite l'expliquer. Cf. aussi R. Barthes, Texte (Théorie du). Encyclopaedia Universalis, corpus 22 p. 370-374, 1973.: «L'intertextualité ne se réduit évidemment pas à un problème de sources ou d'influence; l'intertexte est un champ général de formules anonymes, dont l'origine est rarement repérable, de citations inconscientes ou automatiques, données sans guillemets.»; J. KRisteva, Séméiotikè, Recherches pour une sémanalyse. Paris 1969, 145: «Tout texte se construit comme une mosaïque de citations, tout texte est absorption et transformation d'un autre texte»; P. Sollers, Théorie d'ensemble. Paris 1971, 75: «Tout texte se situe à la jonction de plusieurs textes dont il est à la fois la relecture, l'accentuation, la condensation, le déplacement et la profondeur.» 
au moins selon les exemples de ces genres que nous connaissons, et il ne constitue nullement un récit hagiographique ${ }^{9}$.

Or, étant donné qu'aucune œuvre littéraire ne peut revendiquer une création ex nihilo, indépendante de tout contexte et de toute tradition, il faut certes chercher à détecter la source dans laquelle un élément a été puisé, mais il faudrait aussi chercher la raison pour laquelle il a été utilisé. Il faudrait surtout détecter les changement qualitatifs et peut-être quantitatifs du sens de cet élément mais aussi du texte dans lequel il est inséré c'est-à-dire comment et à quel degré il acquiert et simultanément il procure un nouveau sens à ce contexte. On devrait privilégier le rapport entre le texte-support et le fragment réutilisé au sein du nouvel ensemble formé par leur coexistence. Nous devons considérer que celle-ci est plus qu'une simple juxtaposition ${ }^{10}$, et que l'assemblage de deux textes engendre inévi-

9 Bien que Homère soit discrètement présent dans l'arrière plan de la composition de Digénis, voir p.ex. E, v.712-719 (ed. Alexiou), et bien que Digénis lui-même semble obéir à une morale plutôt épique, voir p.ex. son sens de l'honneur quand il refuse de frapper Kinnamos à terre, E.1279-1280 (ed. Alexiou) et G.VI, 267 (ed. E. Jefrrers, Digenis Akritas, The Grottaferrata and Escorial versions. Cambridge 1998), le thème de l'amour et son rôle dans le développement du récit rend difficile la classification de Digénis parmi les épopées. H.-G. BEck, Formprobleme des Akritas-Epos, in: Beiträge zur Südosteuropa-Forschung. Munich 1966, 137 sq., a même vu dans la première partie du texte de Digénis, le Chant de l'Emir, une épopée et dans la deuxième partie, l'histoire proprement dite de Digénis Akritas, un roman. Pourtant cette bipartition ne répond pas à la réalité du texte puisque dans les deux parties nous pouvons observer une verve épique (scènes de combat, descriptions des guerriers etc.) et des scènes propres aux romans (enlèvement de la femme, scènes sentimentales etc.); voir A. Pertusi, La poesia epica bizantina e la sua formazione: problemi sul fondo storico e la struttura letteraria del Digenis Akritas, in: Atti del Convegno Internazionale sul Tema: La poesia Epica e la sua Formazione. Rome 1970, 481-544. Cependant, il faudrait aussi poser la question de l'unicité du poème, question pertinente qui nécessite une analyse plus approfondie qui n'entre pas dans le cadre de notre approche. Sur les rapports du texte de Digénis Akritas avec l'épopée, le roman et l'hagiographie, voir C. Jouanno, Digénis Akritas, le héros des frontières, Une épopée byzantine. Turnhout 1998, 129-167; plus précisément concernant les rapports du texte de Digénis avec l'épopée et son expression formulaire voir B. Fenik, Digenis, Epic and Popular Style in the Escorial Version. Herakleion-Rethymnon 1991; concernant les rapports du texte de Digénis avec l'hagiographie voir E. Trapp, Hagiographische Elemente im Digenis-Epos. AnBoll 94 (1976) 275-87. Pour l'épisode parallèle de Digénis Akritas et saint Lazare, voir A. Kazhdan, Hagiographical Notes. Byz 54 (1984) 176-192, ici 182, note $\mathrm{n}^{\circ} 6$.

${ }^{10}$ J. Kristeva, La Révolution du langage poétique. Paris 1974, 60: «Le terme d'intertextualité désigne cette transposition d'un (ou de plusieurs) système(s) de signes en un autre; mais puisque ce terme a été souvent entendu dans le sens banal de «critique des sources» d'un texte, nous lui préférons celui de transposition, qui a l'avantage de préciser que le passage d'un système signifiant à un autre exige une autre articulation du thétique - de la positionnalité énonciative et dénotative.» 
tablement une configuration textuelle nouvelle, qualitativement différente de la simple addition de deux unités. Le fragment cité conserve des liens avec son espace d'origine, mais il n'est pas inséré impunément dans un nouveau milieu sans que lui-même et ce nouveau milieu n'en subissent des altérations non négligeables ${ }^{11}$. C'est notamment ce que montre la contribution déjà classique de G. Genette ${ }^{12}$ qui offre de nouvelles perspectives à notre effort de comprendre la littérature byzantine, vu que toute ouvre littéraire peut être considérée comme étant en interaction, directe ou non, avec toutes les œuvres qui l'ont précédée ou qui lui sont contemporaines.

Dans cette perspective, il devient clair que nous devons nous interroger de nouveau sur le sens de la littérature à Byzance et, par la suite, essayer de comprendre son élaboration, son fonctionnement et sa réception par les Byzantins eux-mêmes, tout en prenant en considération que jusqu'au XvIII siècle la doctrine classique de l'imitation était dominante et que ce n'est $q u$ 'à partir du XIX ${ }^{\mathrm{e}}$ siècle romantique que l'idée d'originalité a commencé à peser sur toute la production littéraire ${ }^{13}$.

Dans son histoire presque millénaire, la littérature byzantine se caractérise par un esprit conservateur et réservé. Elle n’a jamais aimé afficher un esprit rénovateur ou innovateur, et encore moins avant-gardiste. Les innovations ou les nouveautés s'inscrivent toujours dans le fil continu des exemples prouvés et approuvés de l'Antiquité et surtout de l'Antiquité hellénistique. Tous les genres de l'Antiquité ${ }^{14}$, et surtout la rhétorique, constituaient indubitablement un terrain fertile où les auteurs byzantins puisaient leur inspiration, et célèbre est leur tendance à les imiter.

Pourtant, il faudrait préciser la notion de mimésis, la façon dont les Byzantins la comprenaient et la pratiquaient ${ }^{15}$. Toute production littéraire

${ }^{11}$ A. Topia, Contrepoints joyciens. Poétique 27 (1976) 353.

12 G. Genette, Palimpsestes, La littérature au second degré. Paris 1982.

${ }^{13}$ Pour une discussion sur la question, voir Influence and Intertextuality in Literary History, ed. J. Clayton - E. Rothstein. Madison Wis. 1991, 4-5, 12-13.

${ }^{14}$ Sur les rapports de la littérature byzantine et la littérature classique voir Byzantium and the Classical Tradition, ed. M. Mullett - R. Sсотт. Birmingham 1981.

15 Sur la question de la mimésis dans le cadre de Byzance, voir H. Hunger, On the Imitation of Antiquity. DOP 23/24 (1969/70) 17-38; A. Kazhdan - G. Constable, People and Power in Byzantium. Washington D.C. 1982, 114-115; La Mimesi Bizantina, in: Atti della quarta Giornata di studi bizantini, a cura di F. Conca e R. Maisano, Milano 16-17 maggio 1996. Naples 1998; Originality in Byzantine Literature, Art and Music, ed. A. R. LitTLEwood (Oxbow Monograph 50). Oxford 1995, et plus précisément, voir les contributions de R. Browning, Tradition and originality in Literary Criticism and Scholarship, p.17-29, R. BEAton, Epic and Romance in the Twelfth Century, p.81-91, ici 88-89 et M. Mullett, Originality in the Byzantine Letter: The Case of Exile, 
doit d'abord et surtout satisfaire la première exigence de la littérature, celle d'être fonctionnelle socialement et culturellement avant de l'être esthétiquement. Ou encore, c'est par sa fonction esthétique qu'elle acquiert et accède à sa fonctionnalité sociale et culturelle. Il ne s'agissait jamais d'une imitation stérile ou d'une reproduction aveugle. L'idée d'un corpus constamment réutilisable, conçu comme réservoir inépuisable d'exemples et de modèles, peut affranchir toute dépendance servile à l'antériorité. Il faut bien le souligner, tout en imitant les anciens, les Byzantins y apportaient des éléments nouveaux aptes à exprimer les nouvelles aspirations de leur réalité socioculturelle. Ainsi, bien qu'un genre équivalant au roman de la seconde sophistique ait vu le jour à Byzance surtout à partir du XII siècle, il en diffère sensiblement ${ }^{16}$ par sa forme même (écrits en vers et non en $\left.\operatorname{prose}^{17}\right)$, par l'emploi qu'il fait de la rhétorique ${ }^{18}$, par l'optique chrétienne qu'il introduit dans l'utilisation des thèmes ou des motifs déjà présents dans le roman hellénistique, comme le thème de l'enlèvement de la femme, le thème de l'Eros ou le motif du jardin ${ }^{19}$.

p.39-58; voir aussi G.T. Dennis, Were the Byzantines Creative or Merely Imitative? BF 24 (1997) 1-9.

${ }^{16}$ R. Beaton, The Medieval Greek Romance. Cambridge 1989; U. Moennig, The late-

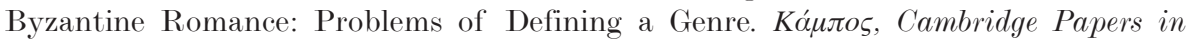

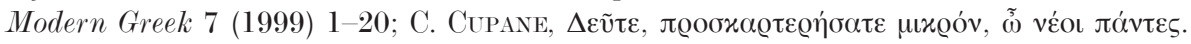
Note sulla ricezione primaria e sul pubblico della letteratura greca medievale. $\Delta i ́ \tau \tau v \chi \alpha$ 6 (1994/95) 147-168; S. MacAlister, Dreams and Suicides. The Greek novel from Antiquity to the Byzantine Empire. London 1996.

17 A l'exception de Makrembolitès, les auteurs byzantins ont préféré composer leurs romans en vers et non en prose. Selon R. Beaton, The Medieval Greek Romance. Op.cit. 72 , cette préférence est due à l'influence de la langue vernaculaire et des romans occidentaux. Mais pour P. Agapitos, Narrative, Rhetoric and 'Drama' Rediscovered: Scholars and Poets in Byzantium interpret Heliodorus, in: Studies in Heliodorus ed. R. L. Hunter (Cambridge Philological Association, Supplementary Volume 21). Cambridge 1998, 125-156, ici 146, elle pouvait être expliquée par l'effort de restaurer la magnifi-

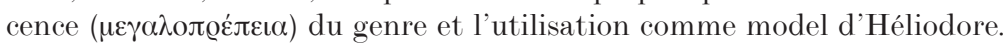

18 P. Rollos, Amphoteroglosia: The Role of Rhetoric in the Medieval Greek Learned Novel, in: Der Roman im Byzanz der Komnenenzeit. Referate des Internationalen Symposiums an der Freien Universität Berlin, 3-6 April 1998, ed. P. Agapitos - D. R. Reinsch (Meletemata 8). Wiesbaden 2000, 109-126.

19 Au-delà de la validité du témoignage de Manuel Philès sur la lecture allégorique d'un roman qui est fort probablement à identifier avec Callimaque et Chrysorrhoé, il est évident que nous devons prendre en considération la présence d'une optique chrétienne, même si elle n'est ni la seule possible ni exclusive des autres, étant donné que la société byzantine productrice de ces textes est profondément chrétienne. Le motif du jardin, à côté de son association à l'Eros, déjà établie depuis Achille Tatius et Longus et exploitée aussi par les auteurs byzantins, devient un motif polysémique pour ces derniers en raison de sa référence implicite, chrétienne et symbolique, au jardin d'Eden et à celui 
Ainsi, pour eux, la phrase clé de Jean Damascène ${ }^{20}$ «je ne dirai rien qui vienne de moi» acquiert un sens nouveau: tout en aspirant à une forme de stabilité qui d'ailleurs, se veut non seulement littéraire mais aussi politique, les intellectuels byzantins revisitent et reprennent les genres de l'Antiquité. Mais ce faisant, ils les font évoluer, voire les recréent en y apportant des changements parfois imperceptibles mais décisifs ${ }^{21}$. C'est le cas de la rhétorique ou du roman qui nous intéresse ici plus précisément. Parallèlement,

du Paradis. Cette possibilité, latente ou patente, de double lecture ne devrait pas échapper aux destinataires de ces récits qui, de surcroît, étaient familiarisés avec la symbolique chrétienne des fleurs (p.ex. la rose pour la Vierge). Sur la question des jardins en tant qu'expression culturelle dans le cadre de Byzance voir: Byzantine Garden Culture, ed. A.R. Littlewood - H. Maguire - J. Wolschke-Bulmahn. Washington D.C. 2002; A.R. LitTlewood, Romantic Paradises: The Role of the Garden in the Byzantine Romance. BMGS 5 (1979) 95-114; H. Maguire, A Description of the Aretai Palace and its Garden. Journal of Garden History 10 (1990) 209-213. Le thème de l'Eros, une constante dans la production romanesque depuis l'antiquité, acquiert dans la nouvelle réalité de ces récits une connotation proprement byzantine. Ainsi, bien qu'Eros soit toujours représenté comme étant un roi/empereur/tyran il ne faut oublier que le terme

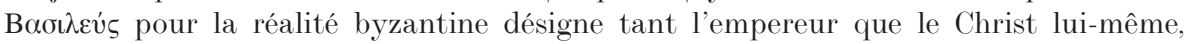
comme le notent P. Agapitos et O. Smith, The Study of Medieval Greek Romance.

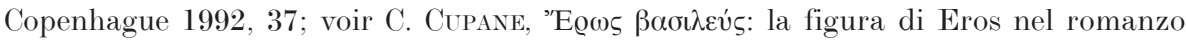
bizantino d'amore. Atti dell' Accademia di Scienze, Lettere e Arti di Palermo. Serie 4, 33/2 (1974) 243-297; ead., Il motivo di castello nella narrativa tardo-bizantina. JÖB 27 (1978) 229-267; H.-G. BEck, Byzantinisches Erotikon. Orthodoxie - Literatur - Gesellschaft (Bayerische Akademie der Wissenschaften, philosopisch-historische Klasse, Sitzungsberichte 5). Munich 1986; P. Magdalino, Eros the King and the King of Amours: Some Observations on Hysmine and Hysminias. DOP 46 (1992) 197-204, où il tient la représentation ailé de l'Eros dans Hysminé et Hysminias pour inspirée des anges à six ailes, hexapteryga, de l'iconographie religieuse; concernant l'œuvre de Makrembolitès et l'utilisation qu'il fait du thème de l'Eros voir I. Nilsson, Erotic Pathos, Rhetorical Pleasure, Narrative Technique and Mimesis in Eumathios Makrembolites' Hysmine and Hysminias. Uppsala 2001, 103-110, 202-208, 279-283. Sur la question de l'amour dans la production littéraire à Byzance il est intéressant de noter que P. ODORICo, L'amour à Byzance. Un sujet de rhétorique? Europe 822 (1997) 34-46, considère qu'il n'y a pas de vraie expression d'amour à Byzance avant l'apparition des romans de la période des Paléologues.

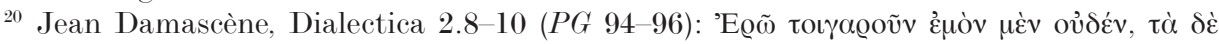

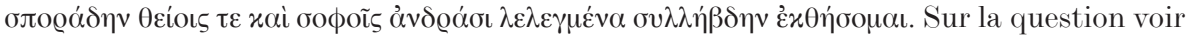

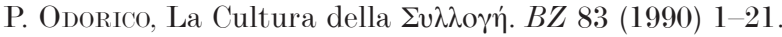

${ }^{21}$ A. Kazhdan et A.W. Epstein, Change in Byzantine Culture in the eleventh and twelfth centuries. Berkeley-Los Angeles-London 1985, 139; A. Kazhdan et S. Franklin, Studies on Byzantine Literature. Op.cit., 185; M. Mulletт, Originality in the Byzantine Letter: The Case of Exile. Op.cit., 40: «... a sense that the Byzantines deny the existence of change, but that change, however slow it may be, surely and perceptibly exists.» 
cette quête de stabilité ou d'imitation ne les empêche pas de créer des genres nouveaux comme les Vies de saints à partir du IV siècle ou les Chroniques Universelles à partir $\mathrm{du} \mathrm{vI}^{\mathrm{e}}$ siècle. Ils mettent ainsi en cause l'imitation aveugle des genres de l'Antiquité dont la création littéraire byzantine a été qualifiée péjorativement et accusée injustement jusque récemment ${ }^{22}$.

Il nous faut alors reformuler la question concernant le roman byzantin et non plus chercher uniquement à trouver les textes qu'il a imités, mais essayer de comprendre pourquoi ce genre n'a pas été repris jusqu'au xII siècle et les raisons pour lesquelles a eu lieu sa réapparition, à quels nouveaux besoins de la société il répondait et comment il a utilisé et réussi à inscrire en lui la donne littéraire des textes antérieurs.

Malheureusement, sur la renaissance des romans, nous ne pouvons que faire des hypothèses qui sont loin d'être reconnues par tous les chercheurs ${ }^{23}$. D'ailleurs, le concept même de «réapparition» doit être mis en doute puisque prétendre que le roman réapparaît au XII siècle équivaut à suggérer qu'il a été complètement oublié et ignoré et qu'il est tombé en désuétude depuis les $I V^{\mathrm{e}}-\mathrm{V}^{\mathrm{e}}$ siècles. Ce que les sources infirment: en Egypte on continuait à les copier jusqu'au VII ${ }^{\mathrm{e}}$ siècle. Photios en mentionne deux dans sa Bibliothèque et selon Psellos les intellectuels de Constantinople débattaient sur la supériorité du roman de Achille Tatius ou d'Héliodore ${ }^{24}$. Nous ne pouvons pas dire, en conséquence, que le roman, après son épanouissement, ait

${ }^{22}$ R. Browning, Enlightenment and Repression in Byzantium in the $11^{\text {th }}$ and $12^{\text {th }}$ centuries. Past and Present 69 (1975) 3-23; B.E. Perry, The ancient romances: A LiteraryHistorical Account of their Origins. Berkeley 1967. Sur la qualification péjorative voir aussi K. Krumbacher, Geschichte der byzantinischen Litteratur von Justinian bis zum Ende des oströmischen Reiches (527-1453) (Handbuch der klassischen Altertumswissenschaft 9.1). Munich ${ }^{2} 1897,767$ : «eine vergröberte und geschmacklose Imitation der nicht sehr geschmackvollen Erzählung des Achilles Tatios von Leukippe und Klitophon»; C. Mango, Byzantine Literature as a distorting Mirror. Oxford 1975, et id., Byzantium. The Empire of New Rome. London 1980; pour une anthologie de ces jugements négatifs voir M. Alexiou, A Critical Reappraisal of Eustathios Makrembolites' Hysmine and Hysminias. BMGS 3 (1977), ici 23-24; P. Agapitos, Narrative Structure in the Byzantine Vernacular Romances. A textual and literary study of Kallimachos, Belthandros and Libistros (MBM 34). Munich 1991, 3-10.

${ }^{23}$ Pour des réponses à cette question, voir Agapitos, Narrative Rhetoric and 'Drama' Rediscovered: Scholars and Poets in Byzantium interpret Heliodorus. Op.cit., 125-156; Beaton, The Medieval Greek Romance. Op.cit., 9-21 et pour des objections à sa thèse voir Agapitos et Smith, The Study of Medieval Greek Romance. Op.cit.; E. Jeffreis, The Comnenian Background to the Romans d'Antiquité. Byz 50 (1980) 455-486; P. Magdalino, Digenis Akritas and Byzantine Literature: The Twelfth-Century Background to the Grottaferrata Version, in: Digenes Akrites, New Approaches to Byzantine Poetry. Op.cit., 1-14.

${ }^{24}$ Cf. note 37. 
disparu pour ne réapparaître qu'au xıI siècle. Les Byzantins connaissaient et lisaient les romans d'autant plus que la production littéraire byzantine ${ }^{25}$ semble s'être appropriée, selon la technique qui lui était si chère, des thèmes et des motifs du roman hellénistique. Il serait donc plus légitime de parler d'une «reprise» plutôt que d'une «réapparition».

Pour pouvoir examiner cette reprise du genre, nous devrions examiner comment les Byzantins eux-mêmes l'ont perçue. Dans cette perspective, au lieu de projeter comme dénominatif de la catégorie générique le terme de roman, et essayer a posteriori de classifier les textes, il faudrait, en paraphrasant l'avertissement de F. de Saussure («C'est une mauvaise méthode

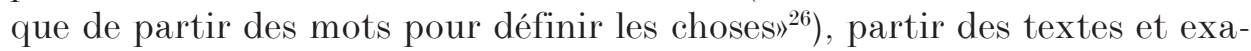
miner les termes utilisés dans leurs titres afin de comprendre à quels types de récit s'attendait le lecteur/auditeur et quelles étaient les caractéristiques du genre auquel ces textes appartenaient.

Pour des raisons purement méthodologiques nous nous limiterons à l'examen des textes qui ont un rapport avec ce que nous considérons aujourd'hui en tant que roman.

Nous ne serions pas étonnés de constater que pour les Byzantins, le terme roman n'existait pas et d'ailleurs, aucune dénomination univoque n'a été utilisée ni par les auteurs ni par les récepteurs de ces œuvres. Ainsi, d'un

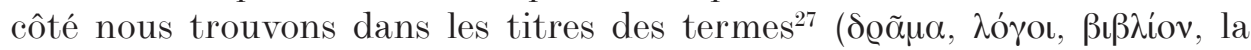

${ }^{25}$ Ainsi, p.ex., Maxime le Confesseur, au viI ${ }^{\mathrm{e}}$ s., utilise dans son sermon des citations de Leucippé et Clitophon et d'Ethiopiques; voir H.-G. BEck, Marginalia on the Byzantine Novel, in: Erotica Antiqua: Acta of the International Conference on the Ancient Novel (ed. B. P. Reardon). Bangor 1977, 59-65, ici 61-62; MacAlister, Dreams and Suicides. Op.cit., 84-114; sur le thème de l'enlèvement de la fille voir Trapp, Hagiographische Elemente im Digenis-Epos. Op.cit., 275-87; sur l'hagiographie voir J. Perkiss, Representation in Greek Saint's Lives, in: Greek Fiction. The Greek Novel in Context (ed. J. R. Morgan and R. Stonemen). London 1994, 223-271; E. Clark, The Life of Melania the Younger and the Hellenistic Romance: A Genre Exploration, in: The Life of Melania the Younger, ed. E. Clark. New York 1984, 153-170, qui en partant de l'ouvrage de R. SödER, Die apokryphen Apostelgeschichten und die romanhafte Literatur der Antike. Stuttgart 1932, explique la spiritualisation progressive qui s'opère à partir du roman grec jusqu'à l'hagiographie chrétienne, en passant par les Actes apocryphes, les Passions des martyr(e)s et les Pseudo-Clémentines; sur l'historiographie voir Ch. RoueCHÉ, Byzantine Writers and Readers: Storytelling in the Eleventh Century, in: The Greek Novel, A.D. 1-1985 (ed. R. BEaton). London 1988, 123-133.

${ }^{26}$ F. De Saussure, Cours de linguistique générale. Wiesbaden 1968.

${ }^{27}$ Il est à souligner que les appellations des «romans» varient, pour une même œuvre, d'un manuscrit à l'autre et que le titre inscrit sur un manuscrit n'est pas nécessairement de l'auteur. 


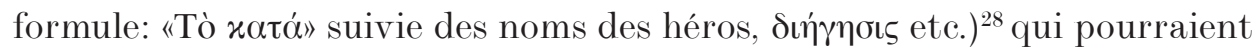
bien être utilisés pour d'autres sortes de récits, comme par exemple l'hagiographie ou les exercices rhétoriques, et, de l'autre côté, nous trouvons,

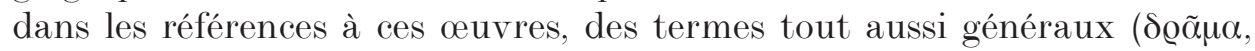

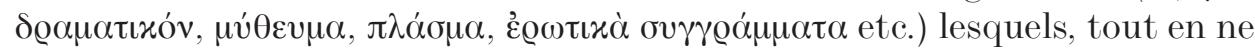
dénotant pas une catégorie littéraire spécifique, mettent cependant en évidence la nature du récit et laissent apparaître les clauses du pacte qui unissent l'auteur à ses récepteurs. Dans cette perspective, le «roman» s'affiche clairement comme une intrigue fictive, donc construite, régie par un pacte qui est celui de l'illusion ${ }^{29}$.

Or, tous ces termes, et cela malgré leurs différences, appartiennent à un fond sémantique commun et indiquent plutôt un procédé d'écriture qu'un genre littéraire particulier, signe révélateur, peut-être, d'un genre qui, depuis son apparition, n'a jamais su trouver un défenseur, tel Aristote pour la tragédie, qui l'aurait défini comme tel et lui aurait conféré ses lettres de noblesse. Il s'agit de termes dont la fonction est de prendre en charge la mise en scène textuelle de l'acte de raconter une histoire adoptant, la plupart des fois, la structure d'une biographie, d'un récit de vie. Par conséquent, le terme de narration est le terme général qui rassemble toutes ces notions.

Ainsi, pour les Byzantins, destinataires de ces œuvres, indépendamment du fait qu'il s'agissait du cercle fermé d'hommes de lettres ou non, ces œuvres appartenaient à une catégorie littéraire précise et elles obéissaient à un code plus ou moins strict et à une structure plus ou moins souple qui leur permettaient de se former selon leur propre prérogative et leur propre sens. Par conséquent, elles présentaient des traits communs qui étaient donc détectables et compréhensibles par leur récepteurs.

${ }^{28}$ Pour une liste des termes utilisés dans les titres des «romans» mais aussi dans les prooimia, voir aussi Agapitos, Narrative Structure in the Byzantine Vernacular Romances. Op.cit., 43-45. Il est intéressant de noter que P. Agapitos examine aussi la question des termes utilisés par des auteurs tels Photios, Psellos, l'empereur Julien ou même Plutarque quand ils se réfèrent à cette catégorie de la production littéraire et il note, p.43: «The novel ... did not receive a specific genre name ... we find expressions like $\pi \lambda \alpha \dot{\sigma} \mu \alpha$

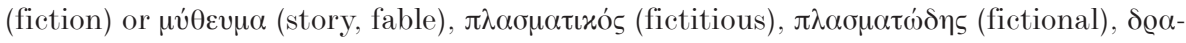

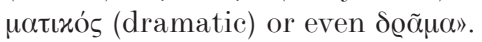

${ }^{29}$ Nous tenons que c'est exactement à ce pacte d'illusion réaliste que fait référence Photios quand dans son commentaire concernant Les merveilles incroyables d'au delà de Thulé



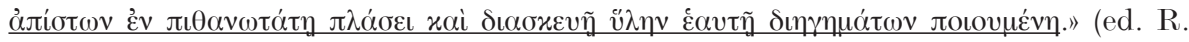
Henry, Photius Bibliothèque. Paris 1951-1991, 166.109a10-12) (c'est nous qui soulignons). 
Or, le terme de narration indique une catégorie qui présente l'avantage d'être plus élargie que celle du roman ou du 'romance' ou 'novel' pouvant englober tant les romans de la période comnène $\left(\mathrm{XII}^{\mathrm{e}} \text { siècle }\right)^{30}$ que Digénis Akritas $^{31}$, les romans de la période des Paléologues (XIV siècle) ${ }^{32}$, l'Achilléi$\mathrm{de}^{33}$, le roman d'Alexandre ${ }^{34}$, l'histoire de Bélisaire ${ }^{35}$ ou même la Narration de la guerre de Troie ${ }^{36}$ et d'autres. On voit donc, à côté des quatre ouvres appelées «romans» byzantins du XII siècle, une suite d'autres textes qui ne partagent avec eux ni le même degré de stylisation ni la même marque d'imitation ou d'influence des ouvres de la seconde sophistique ${ }^{37}$. Tous ces textes avaient pour les Byzantins des traits communs suffisants pour qu'ils

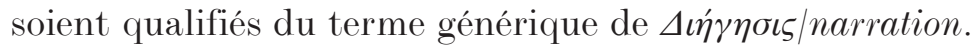

${ }^{30}$ Drosilla et Chariclès de Nicétas Eugénianos (ed. F. Conca, Nicetas Eugenianus De Drosillae et Chariclis amoribus. Amsterdam 1990); Hysminé et Hysminias de Eustathios Makrembolitès (ed. M. Marcovich, Eustathius Makrembolites De Hysmines et Hysminiae amoribus libri XI. Munich-Leipzig 2001); Aristandros et Kallithéa de Constantin Manassès (ed. O. Mazal, Der Roman des Konstantinos Manasses. Überlieferung, Rekonstruktion, Textausgabe der Fragmente [WBS IV]. Vienne 1967; E. Tsolakes,



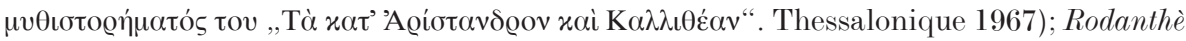
et Dosiklès de Théodore Prodrome (ed. M. Marcovich, Theodori Prodromi De Rhodanthes et Dosiclis amoribus libri IX. Stuttgart 1992); voir aussi Il romanzo bizantino del XII secolo (ed. F. Conca). Turin 1994, qui a édité l'ensemble des romans de la période comnène.

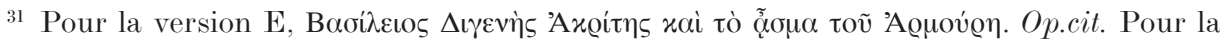
version G, Jefrreys, Digenis Akritas, Op.cit. Sur les versions E, G, Z voir Digenes Akrites: synoptische Ausgabe der ältesten Versionen, ed. E. Trapp (WBS VIII). Vienne 1971.

32 Callimaque et Chrysorrhoé (ed. M. Pichard, Le Roman de Callimaque et Chrysorrhoé.



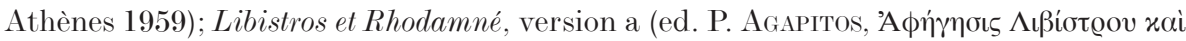


et Blancheflor) (ed. Kriaras, op.cit.); Imperios et Margarona (ed. Kriaras, ibidem). Pour les romans de la période des Paléologues voir aussi l'édition de C. Cupane, Romanzi cavallereschi bizantini. Turin 1995 .

${ }_{33}$ O. Smith, The Byzantine Achilleid. The Naples Version, édition posthume par P. AGAPITOS - K. Hult (WBS XXI). Vienne 1999, avec en appendice la version d'Oxford.

${ }^{34}$ W. Wagner, Trois Poèmes Grees du Moyen-Age. Berlin 1881.

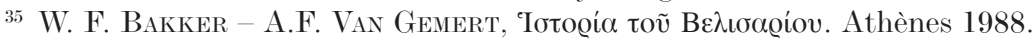

${ }^{36}$ L. Norgand - O. Smith, A Byzantine Iliad. The text of Par. Suppl. Gr. 926. Copenhagen 1975 .

${ }^{37}$ Nilsson, Erotic Pathos. Op.cit., 19 sq. a noté que la relation entre Achille Tatius et les romans de la période d'avant la deuxième sophistique est analogue à celle entre Makrembolitès et Tatius, c'est-à-dire, empreinte de certains éléments réutilisés dans un contexte nouveau et de liens intertextuels avec d'autres genres littéraires. 
Dans cette perspective et après un examen, certes non exhaustif, de l'ensemble de ces ouvres, nous pouvons constater que les Byzantins ont invoqué presque tous les genres littéraires, antiques et contemporains ${ }^{38}$, de l'épopée à l'historiographie, des exercices de rhétorique à la comédie nouvelle, comme si le «roman» était né de la désagrégation de toutes les pratiques poétiques et rhétoriques codifiées. Comme si le «roman» avait utilisé et refondu dans sa structure quasiment tous les genres de la littérature!

Toutes les œuvres que nous avons évoquées plus haut partagent un élément structurel de base: la narration, soit de l'histoire d'une vie, soit d'un moment de cette vie. Certes d'une manière différente, mais ils la relatent parce qu'elle a eu quelque chose de mémorable, dans son ensemble ou juste en partie, et qu'elle a eu surtout quelque chose de merveilleux qui la rapproche du mythe.

Il s'agit bien de la narration de l'histoire d'une vie, partielle ou non, d'une vie imaginée ou même inspirée de la réalité historique ou contemporaine de l'auteur avec laquelle elle entretient des liens très lâches, adressée à un public averti, voire prévenu, en quête plutôt d'une lecture plus légère, peut-être moralisante mais surtout divertissante ${ }^{39}$, au sens étymologique

${ }^{38}$ Nous pourrions observer le même processus constaté par E. Rohde concernant le roman hellénistique, E. Roнde, Der griechische Roman und seine Vorläufer. Wiesbaden 1867. Selon lui, la naissance du roman aurait été déterminée par la fusion de l'élégie érotique alexandrine et des récits d'aventures de voyage. Pourtant, chez les Byzantins ce processus de fusion/mixtion ne constitue pas une particularité propre du roman mais, au revanche, concernait toute la production littéraire. Sur cette question voir MULLETT, The Madness of Genre. Op.cit.; P. Agapitos, Mischung der Gattungen und Überschreitung der Gesetze: Die Grabrede des Eustathios von Thessalonike auf Nikolaos Hagiotheodorites. JÖB 48 (1998) 119-146.

39 Agapitos - Smith, The Study of Medieval Greek Romance. Op.cit., 22, ont émis des objections sur l'utilisation du terme «divertissante», dont la paternité est attribué selon les auteurs à H.-G. BEck. Pourtant, tout en reconnaissant, comme eux, le besoin d'une étude plus approfondie sur «the literary public in Byzantium», id., note 36, nous utilisons ce terme parce que nous croyons que dans ce type de récit, le lecteur byzantin n'y cherchait pas le même effet (p.ex. moralisant ou édifiant) qu'il aurait trouvé plus facilement, parce que plus directe et explicite, dans un autre type de récit (comme p.ex. une vie de saint, un discours rhétorique ou une historiographie). Nous ne contestons pas le côté «divertissant» que la lecture de ces types de texte peut provoquer mais nous tenons que le pacte littéraire entre l'auteur et le lecteur dans le cas de l'hagiographie, de la rhétorique ou de l'historiographie est d'une nature tout autre que dans le cas de récit de vie que nous venons d'évoquer. Sur la question de la perception ou de la lecture allégorique des romans, les témoignages de Photios, Bibliothèque 73.50a6, 87.66a14, 94.73b35 et 166.109a6 (ed. R. Henry, Photius Bibliothèque. Paris 1951-1991), de Psellos (ed. A. R. Dyck, The Essays on Euripides and George of Pisidia and on Heliodorus and Achilles Tatius [BV 16]. Vienne 1986), de Manuel Philès (E. Martini, A proposito 
du terme. Il s'agit donc de raconter une histoire «réelle» mais pas vraie. L'historiographie raconte une histoire que l'auteur autant que le récepteur (auditeur ou lecteur) croient réelle et dont les faits, ainsi que les personnes mentionnées, sont perçus comme étant vrais. Le roman, de son côté, raconte lui aussi une histoire mais le pacte avec le récepteur/auditeur étant d'une toute autre nature, l'histoire racontée n'a plus besoin d'être vraie mais il lui suffit d'être conforme à la vérité ${ }^{40}$.

Une histoire du type in eo tempore. Une histoire qui, sous le prétexte du hic et nunc, se réfère à un ailleurs et à ces temps-là tout aussi indéterminables que ses héros: tout en évoluant à travers un espace ou même un temps quelque part familier, tout en utilisant un vocabulaire se référant à des realia ${ }^{41}$, tout en se conformant plus ou moins aux normes de la société, ces héros sont néanmoins dépourvus d'une entité au sens existentiel du terme, étant donné que la littérature ${ }^{42}$ constitue leur seule réalité, et se transforment en figures «mythiques» ${ }^{43}$.

d'una poesia inedita di Manuele File. Rendiconti del Regio Istituto Lombardo di Scienze e Lettere, ser. II/29. Milan 1898,460-465), se révèlent d'une valeur inestimable étant donné qu'ils sont les seuls que nous possédions de la période. Nous pouvons aussi extraire quelques renseignements indirects sur la diffusion des romans par le contenu des bibliothèques comme p. ex. celle de Eustathios Boilas, voir S. VRYonis, The will of a provincial magnate: Eustathios Boilas (1059). DOP 11 (1957) 263-277. Sur cette question voir P. ODorico, La circulation des livres en Italie du Sud $\mathrm{X}^{\mathrm{e}}-\mathrm{XII}^{\mathrm{e}}$ siècles. Une originalité?, in: L’Ellenismo Italiota dal vII al XII secolo. Alla memoria di Nikos Panayotakis (Istituto di Ricerche Bizantine. Convegno Internazionale 8). Athènes 2001; BEck, Marginalia on the Byzantine Novel. Op.cit., 59-65; Agapitos, Narrative, Rhetoric and 'Drama' Rediscovered: Scholars and Poets in Byzantium interpret Heliodorus. Op.cit., ici $133-137$.

${ }^{40}$ Cf. note 28. Voir aussi B. E. Perry, The Ancient Romances: A Literary-Historical Account of Their Origins. Berkeley - Los Angeles 1967, 10: «... historiography cannot become romance without passing through zero, that is, through the negation of its own raison d'être, that which defines it as historiography».

${ }^{41}$ R. BARThes, dans son fameux article «L'effet de réel». Communications 11 (1968), repris dans R. Barthes - L. Bersani - P. Hamon - M. Riffaterre - I. Watt, Littérature et réalité. Paris 1981, 81-90, montre bien qu'un certain nombre d'éléments présents dans le texte réaliste ne dénotent en rien le réel, mais qu'ils sont là pour connoter le réalisme, pour susciter un effet de réel et produire chez le lecteur l'illusion réaliste ou référentielle. Voir aussi H. Hunger, Un roman byzantin et son atmosphère: Callimaque et Chrysorrhoé. TM 3 (1968) 405-22.

${ }^{42}$ Dans le cadre de cette discussion, le terme littérature se réfère à la littérature tant orale qu'écrite. Sur la question de la littérature orale voir R. Beaton, Orality and the Reception of Late Byzantine Vernacular Literature. BMGS 14 (1990) 174-184.

${ }^{43}$ Pour une analyse plus approfondie de la fonction du mythe voir entre autres, R. Trousson, Servitude du créateur en face du mythe. Cahiers de l'Association Internationale des Etudes Françaises 20 (1968) 85-98; A. J. Greimas, Eléments pour une théorie de l'inter- 
Ainsi, de grandes figures de l'histoire et même de la tradition byzantine ont atteint, par leur fortune et leur portée significative, la valeur de mythe littéraire, depuis bien longtemps dépouillée de son contenu historique d'origine. Elles sont la représentation symbolique d'une situation humaine exemplaire, d'un cas particulier haussé à la proportion universelle afin de pouvoir répondre aux besoins et aux espérances de la société qui a vu naître ces figures. A la linéarité historique, alors, succède la vision kaléidoscopique du mythe ${ }^{44}$.

Donner vie à un personnage de roman, c'est d'abord lui fournir une épaisseur par les moyens qui sont ceux de la littérature, c'est-à-dire moins la psychologie ou l'histoire que la littérature elle-même. Le nom ${ }^{45}$ est alors une donnée essentielle, qu'il soit intertextuel, comme Alexandre ou Achille, ou qu'il élabore autour de lui un faisceau de traits distinctifs nouveaux comme Digénis Akritas, Hysminias ou Dosiclès. Inscrire des noms ou des caractères qui renvoient explicitement à la littérature antérieure, c'est à la fois attester la présence d'un personnage existant, pourvu d'attributs connus et imposer un système de références autonomes.

La constitution d'un espace-temps spécifique au récit exige la mise en place de certains éléments susceptibles d'entraîner la reconnaissance du lecteur. Cette mise en place se réalise le plus souvent à l'aide d'un montage entre données descriptives et données livresques. Si l'évocation de la réa-

prétation du récit mythique. Communications 8 (1966) 29; P. Albouy, Mythes et mythologies dans la littérature française. Paris 1969, 293; D. Anzieu, Freud et la mythologie. Incidences de la Psychanalyse I (1970) 124.

${ }^{44}$ B. D. Macqueen, Myth, Rhetoric and Fiction, A reading of Longus's Daphnis and Chloe. Lincoln and London 1990, xi: «This concession, which amounts to some sort of tacit agreement between the author and the reader that the incidents and characters about to be described are not real, allows the novelist to aim at another kind of truth, of a sort that can never, or with difficulty, be reached through the discursive representation of reality."

45 Sur les noms propres M. DE Certeau (L'écriture de l'histoire. Paris 1975, 309) note: «De toute façon, la dénomination pose un lien en même temps qu'un lieu. Elle fonctionne à la fois comme participation à un système et comme accès au symbolique»; voir aussi C. Lévi-Strauss, La pensée sauvage. Paris 1962, 245: «les noms propres sont toujours significatifs de l'appartenance à une classe actuelle ou virtuelle qui peut être seulement celle de celui qu'on nomme ou celle de celui qui nomme» et p. 285-286: «le nom propre demeure toujours du côté de la signification». Pour un point de vue contraire voir A. H. Gardiner, The Theory of Proper Names. A controversial Essay. Londres ${ }^{2} 1954$, qui soutenait l'insignifiance des noms propres et opposait désignation à signification. Cf. aussi J. R. Searle, Proper Names, in: J. F. Rosenberg - C. Travis, Readings in the Philosophy of Language. Prentice-Hall 1971, 212-218. 
lité byzantine est aussi forte dans ces récits, c'est qu'on y reconnaît plusieurs espaces et plusieurs temps.

Les héros de ce genre ou mixtion littéraire, comme par exemple Digénis Akritas, partagent avec les autres héros de ce type de récit, Alexandre, Achille, Bélisaire mais aussi Hysminias, Dosiclès, Libistros ou Callimaque, des caractéristiques communes; lesquelles sont, avant tout, des caractéristiques structurelles de la catégorie dont ils font partie ${ }^{46}$. L'ensemble des propriétés structurelles communes à tous ces récits constitue un modèle narratif reconnaissable par les Byzantins, et ce modèle doit rendre compte à la fois du récit considéré comme unité discursive transphrastique et de la structure du contenu qui est manifesté au moyen de cette narration ${ }^{47}$. Ces caractéristiques se construisent chaque fois différemment afin de former un récit qui répond à ses propres prérogatives et qui véhicule son propre sens $^{48}$.

Quelles sont ces caractéristiques? Le récit se construit autour d'une histoire de vie d'un héros qui est porteur d'un destin singulier et extraordinaire, qu'il doit réaliser et confirmer à travers une suite d'épreuves. Ce destin pourrait être de conquérir le monde, de se marier avec sa bien-aimée, ou tout simplement de prouver qu'il est un héros.

Ainsi, tous sont des héros dont la vie et la prouesse conditionnent toute l'histoire du récit, qui d'ailleurs se structure uniquement autour d'eux, autour de leurs exploits, de leurs amours ou des deux. Ils doivent être jeunes, beaux et surtout courageux pour pouvoir endurer les mille péripéties qu'ils affrontent. Parce que le thème de base de toutes ces narrations, n'est que le combat du héros contre le temps et l'espace afin de réaliser son propre désir, voire pour se réaliser, se confirmer en tant que héros.

Or, nonobstant leurs éléments structuraux communs, toutes ces figures ne sont ni similaires ni identiques.

46 Ces héros partagent aussi quelques-unes de ces caractéristiques avec d'autres figures de la production littéraire byzantine, comme celles des saints. La différence, pourtant, est que les saints sont des figures qui appartiennent à la sphère du sacré, et en conséquence, ils se trouvent investis d'un credo qui leur apporte aussitôt une réalité indiscutable dépassant largement le cadre de la littérature.

${ }^{47}$ Greimas, Eléments pour une théorie de l'interprétation du récit mythique. Op.cit., 35.

48 Anzieu, Freud et la mythologie. Op.cit., 124: «un mythe est un récit composé par un enchaînement de phrases fondamentales distinctes; ces mythèmes ou éléments mythiques de base sont communs à plusieurs mythes, un mythe particulier se caractérise par le choix des mythèmes et par la façon de les organiser.» 
A ce stade, nous pourrions essayer une première distinction de ce type de récit en deux sous-catégories ${ }^{49}$ :

a. Le premier type est le «récit de vie partielle». Il s'agit d'un récit à deux protagonistes $^{50}$, quoique la figure du héros soit effectivement plus présente et même plus pesante que celle de l'héroïne ${ }^{51}$. Il s'agit d'un récit à «thème de situation", où la narration absorbe les héros qui se définissent comme tels par rapport à un ensemble en soi immuable sur le plan des circonstances. La signification du thème est conditionnée par un nœud de données caractérisant; les personnages n'existent, dans leur portée symbolique, que par cet ensemble dont ils sont une fonction. L'amour du héros pour l'héroïne constitue le motif, voire le mobile par excellence du thème à un tel degré qu'il absorbe leurs traits différentiels, et les héros, réduits à des figures interchangeables avec les figures des autres récits du même type, se voient dépendre exclusivement du destin de cet amour.

b. Le second type est celui du «récit de vie intégrale» d'un seul protagoniste qui dépasse la situation, la fait contingente ou la crée. C'est un récit

49 R. Beaton, Epic and romance in the twelfth century, in: Originality in Byzantine Literature (ed. A. R. LitTLEWOOD). Op.cit., 84, a proposé une distinction entre récits à un seul héros qui constitueraient le récit épique et récits à deux héros qui constitueraient le récit romanesque. Nous ne partageons pas cette catégorisation, parce que pour nous l'existence même de l'épopée à Byzance est incertaine et problématique et que de plus tous ces textes partagent un très grand nombre d'éléments structuraux qui nous incitent à les considérer comme faisant parti d'une même catégorie. A l'instar de R. Beaton, C. Cupane, Roman. VII. Byzantinische Literatur. LexMA VII 988-990. Munich 1996, propose une distinction entre romans originaux et adaptations des romans occidentaux. Moennig, The late-Byzantine Romance: problems of defining a genre, op.cit., propose une catégorisation selon deux critères: celui du temps, voire le temps de l'aventure ou le temps biographique, et conséquemment celui du développement du thème de l'amour ou du thème de l'histoire. Nous objectons à cette catégorisation de ne pas prendre en considération le pacte qui détermine tant l'intention signifiante de l'auteur que l'attente du public ni, par conséquent, le fait que la narration n'utilise le thème de l'histoire que comme thème adjuvant, un arrière-plan, afin de déployer le récit porteur d'un sens qui n'est nullement historiographique. Bélisaire, en constitue à nos yeux l'exemple le plus significatif. Sur cette question, voir aussi MacQueEn, op.cit., 138-160.

${ }^{50}$ Parfois, nous trouvons un deuxième couple dont le récit «secondaire» est inséré dans le récit «principal», et que G. Genette nomme «récit métadiégétique». Mais ce récit n’a qu'une fonction «adjuvante» et n'acquiert jamais l'importance ou le développement du récit principal.

${ }^{51}$ Les deux protagonistes bien qu'ils soient héros au même titre, ont un rôle dans le récit différencié afin de mieux s'adapter aux exigences sociales des récepteurs. Ainsi par exemple le héros est plus présent et actif que l'héroïne qui tient un rôle plus passif comme c'est le cas, par exemple, dans Rodanthè et Dosiclès où c'est par l'intermédiaire du héros que nous apprenons l'histoire de la fille. 
à «thème de héros». Sa polyvalence et son autonomie le mettent à l'abri d'une fixité. Elles lui assurent non seulement une complète indépendance, mais aussi et surtout un sens symbolique. Celui-ci est résumé dans le héros, quelles que soient son action et les circonstances dans lesquelles la fantaisie du poète choisira de le faire évoluer. Dans ce récit, on rencontre aussi le motif de l'amour pour une femme qui, loin de constituer le motif principal, est confiné à un rôle adjuvant. La femme, dès lors, se voit reléguée à un rôle secondaire par rapport au héros. D'ailleurs, nous ignorons la plupart des fois jusqu'au nom de la femme.

Ainsi, d'un côté nous percevons des héros tels Hysminé et Hysminias, Drosilla et Chariclès, Libistros et Rhodamné ou Callimaque et Chrysorrhoé. Ces héros forment la sous-catégorie «récit de vie partielle» dont nous suivons le récit d'un fragment de leur vie et plus précisément, juste le récit de leur amour. Ils sont déjà nés quand la narration commence et rien ne nous est raconté sur leur mort. Ce qui déclenche la narration, c'est le fait qu'ils tombent amoureux ${ }^{52}$. Le temps et l'espace à travers lesquels ils défilent se trouvent eux aussi fragmentés, étant donné que ce qui intéresse la narration n'est ni l'avant ni l'après de cette histoire ni le lieu où elle se déroule mais la situation. Le personnage ne se définit comme symbolique et représentatif que par la situation. Ainsi, tout commence quelque part, à un certain moment: lors d'un voyage pendant les fêtes religieuses Diasia pour Hysminé et Hysminias; alors que les pirates attaquent l'île de Rhodes et emprisonnent Dosiclès et Rodanthé; pendant une invasion des Barbares en Thrace dans le cas de Drosilla et Chariclès; dans un pays où vivait un roi qui avait trois enfants... pour Callimaque et Chrysorrhoé. C'est dans ce fragment spatio-temporel que se développera tout le récit, que la situation sera déployée à travers leurs aventures extraordinaires, la rencontre, l'enlèvement, l'obstacle à leur amour, l'esclavage, vers un dénouement heureux: la réunion et le mariage. C'est le récit d'un fragment d'une vie fictive, et les liens qu'il entretient avec la réalité sont vagues. Ce qui importe, dans ce genre de narration, n'est pas son ancrage dans la réalité mais le thème développé par la narration.

De l'autre côté, nous trouvons des héros tels Digénis Akritas, Alexandre, Achille ou Bélisaire. Des héros qui partagent de surcroît un arrière-plan

${ }^{52}$ Bien que l'histoire commence, dans le cas de Callimaque et Chrysorrhoé, avant que les deux héros tombent amoureux, pourtant, l'amour reste toujours le moteur de la narration. Ainsi, jusqu'au v. 450, où le héros rencontre pour la première fois la fille, la narration nous présente les raisons qui ont amené notre héros à s'aventurer jusqu'au Château du dragon: son père, le roi, a décidé de choisir comme héritier celui qui accomplira le plus grand exploit. 
historique réel ou au moins d'apparence historique. Pourtant, une fois en situation de récit, cet arrière-plan se dissout, et les indices du temps ou de l'espace, de même que toute empreinte d'une époque, deviennent flottants et indécis. Subséquemment, le thème du héros, très tôt séparé d'un contexte fixe, d'un cadre défini, acquiert spontanément une polyvalence et une multiplicité de significations. Ainsi nous suivons le récit de vie de ces héros depuis leur naissance jusqu'à leur mort à travers leurs combats, leurs amours, leurs destins, mais chacun d'entre eux se voit investi d'un sens différent. Le langage réel du texte renvoie toujours à un autre langage, langage virtuel, à l'horizon de la figure, qui envisage le lecteur-interprète.

Digénis Akritas est un héros qui évolue dans un espace et un temps imprégnés d'indications historiques qui, toutefois, restent imprécises et indéterminées. De ce fait, nous ne pouvons le situer dans la réalité historique de Byzance, bien que nous puissions le situer dans une réalité byzantine qui est la réalité imaginaire et abstraite appréhendée par les Byzantins eux-mêmes, voire la projection de la réalité sur le plan littéraire. Dans cette perspective, l'on rencontre dans la généalogie de Digénis Akritas des noms de familles aristocratiques précis, comme celui des Doukas ${ }^{53}$ ou imprécis comme celui des Kirmagistroi ${ }^{54}$ ou encore des noms arabes comme Mourstasit ${ }^{55}$ ou Mousour ${ }^{56}$. Pourtant, ces noms, plus qu'à des familles ou à des personnalités réelles, renvoient surtout à une généalogie crédible en rapport avec les connaissances et les attentes des récepteurs. Digénis Akritas rencontre des adversaires réels, avec une entité historique réelle, tels les Apélates, ou des adversaires imaginaires, tels Maximô ou le dragon: ce qui importe n'est pas l'adversaire en lui-même, mais ce qu'il représente dans l'imaginaire de la société byzantine.

Alexandre évolue dans un espace qui est plus mythique que réel et dans un temps qui est dilaté par l'incorporation dans le récit des événements historiques mais aussi des anachronismes et des affabulations ${ }^{57}$. Le nom des

${ }^{53}$ E 137 (Alexiou). G 1.267; 4.43; 4.59; 4.325; 6.14; 6.414 (JefFreys).

54 E 138 (Alexiou).

55 E 261 (Alexiou).

${ }_{56}$ E.723. G 5.168; 5.203; 5.215; 5.261 (Alexiou).

${ }_{57}$ Pour une étude approfondie du Roman d'Alexandre voir C. Jouanno, Naissance et métamorphose du Roman d'Alexandre. Paris 2002. Plus précisément, sur l'utilisation du temps historique par le roman voir le ch. III, «Le Roman et la tradition historique», p.128-189, où l'auteur note, p. 144: «La réalité historique apparaît donc dans le Roman à travers un prisme déformant: on peut y reconnaître maints épisodes authentiques de la geste d'Alexandre, mais désordonnés à l'intérieur d'une configuration nouvelle» pour continuer p.153: "quoiqu'elles contribuent au processus de mythification, les diverses altérations que l'auteur du Roman fait subir à l'histoire d'Alexandre tiennent sans 
pays qu'il conquiert, quand ils ont existé historiquement, est investi d'une aura irréelle qui ne laisse aucune trace de leur réalité socio-politique, comme cette Egypte tant pharaonique qu'hellénistique et magique. Dans le cas contraire, il s'agit de pays mythiques, comme celui des Amazones ou le pays des Cynocéphales, ou quasi-mythiques, comme celui de l'Inde qui marque les limites lointaines de l'œecoumène, de l'univers ${ }^{58}$. Mais c'est exactement ce détachement de la réalité historique qui lui confère sa caractéristique principale: c'est un héros avant tout conditionné par son destin historique qu'il doit prouver encore une fois en le transférant dans la réalité byzantine ${ }^{59}$ et c'est dans ce sens qu'il réalise tous ses combats légendaires afin de conquérir le monde et de prouver qu'il mérite bien ce que le destin lui a réservé.

Achille évolue lui aussi dans un espace-temps non déterminé, le seul indice étant que son père, un des rois grecs, était le roi des Myrmidons ${ }^{60}$. Ses liens avec la réalité byzantine ou même avec le texte homérique restent aussi lâches que ceux des autres héros que nous venons d'évoquer, et dépendent surtout des noms mentionnés par le récit et un vocabulaire qui renvoie à des realia (p.ex., $\mu \alpha \tilde{v} \varrho o^{61}, \mu \varepsilon \tilde{\varrho} \alpha \xi^{62}$, $\tau$ ovßíov ${ }^{63}$ etc.) Ses combats et

doute dans une large part à l'incertitude de ses connaissances... d'ailleurs peu surprenantes dans une œuvre qui repose tout entière sur un anachronisme...» Pourtant, selon nous, «les diverses altérations que l'auteur du Roman fait subir à l'histoire d'Alexandre» sont moins dues «à l'incertitude de ses connaissances» qu'à son intention signifiante, laquelle est de composer non pas un récit historique de la vie d'Alexandre mais un récit qui transgresse précisément l'histoire pour viser Alexandre le symbole, conformément au pacte conclu entre l'auteur et ses récepteurs et dans le respect de ces clauses. Cf. infra p. 227. et aussi note 66 .

${ }^{58}$ L'intérêt de s'interroger sur les sens de cette géographie pour les Byzantins n'est pas niable. Etait-elle tellement mythique pour eux? Tous ces noms de pays, de villes, de contrées que traversent les héros, reviennent fréquemment dans les œuvres de géographie, les romans, l'histoire ou les vies de saints. Sur la question de la géographie voir, P. Odorico, L'uomo nuovo di Cosma Indicopleuste e di Giovanni Malalas. BSl LVI (1995) 305-315.

${ }^{59}$ Concernant l'importance d'Alexandre pour la société byzantine, voir la thèse de N.S. Trahoulia, A study of Alexander the Great as an Imperial Paradigm in Byzantine Art and Literature (Department of Fine Arts, Harvard University). Cambridge, Massachusetts 1997.

${ }^{60}$ La remarque de Smiтh, The Byzantine Achilleid, The Naples Version. Op.cit., 83: «In Byzantium the word Myrmidons was used about Bulgarians; at least this is the only meaning of the word outside Homeric contexts» est très intéressante, mettant exactement l'accent sur l'utilisation des termes par les Byzantins en tant que signes à signifié flottant.

${ }^{61} \mathrm{~N} 134,377$ (Sмптн).

${ }^{62}$ N108 (Sмiтh).

${ }^{63} \mathrm{~N} 1199$ (Ммітн). 
ses aventures menés contre des adversaires anonymes l'affirment en tant que Héros mais c'est surtout en combattant l'Eros éprouvé envers et de par la femme qu'il confirme sa position.

Bélisaire se voit lui aussi évoluer dans cet amalgame spatio-temporel qui fait de Justinien et des Paléologues des contemporains et introduit une guerre contre l'Angleterre pour évoquer celle contre les Vandales. La réalité historique de Bélisaire perd de son importance pour qu'il puisse devenir ce héros qui a su protéger l'Empire contre ses ennemis et qui, en même temps, a su réaliser son destin et se confirmer en tant que héros exceptionnel.

Ces héros forment la deuxième sous-catégorie «récit de vie intégrale», voire récit biographique. C'est le récit d'une vie qui se veut réelle et authentique mais qui se trouve privée de ses attributs qui la liaient autrefois à la réalité historique. Les péripéties aventureuses n'ont aucun lien matériel avec les particularités des pays qui y figurent, avec leur structure sociopolitique, leur culture, leur histoire. Aucun de ces caractères particuliers n'y entre en qualité d'élément déterminant. Ce qui importe, dans ce genre de narration, n'est pas son ancrage dans la réalité, mais le développement narratif de la figure littéraire du héros qui se trouve seul porteur du sens de la narration. Il serait intéressant de noter pourtant que le récit de Bélisaire côtoie dangereusement les récits de vie partielle. D'un côté, il est un héros seul qui évolue dans l'espace et le temps à la manière des autres héros de la catégorie du récit de vie intégrale. De l'autre côté, derrière cette évolution, il y a un motif concret, une cause principale, l'envie qui, à l'instar de l'Eros de la catégorie de récit de vie partielle, constitue le mobile de la narration. Cependant, le thème de l'Eros n'entre pas dans le déploiement du récit et l'œuvre ne s'achève pas sur un mariage heureux mais, bien au contraire, par un aveuglement malheureux équivalant à la mort politique et sociale du héros. De surcroît, la différence la plus frappante entre ce récit et ceux de la catégorie de vie partielle est que Bélisaire constitue un récit à un seul protagoniste qui ne peut pas être remplacé par un autre héros. L'histoire narrée appartient à Bélisaire et à lui seul, non seulement en raison de son ancrage historique réel mais aussi et surtout en raison de sa récupération par l'imaginaire byzantin ${ }^{64}$.

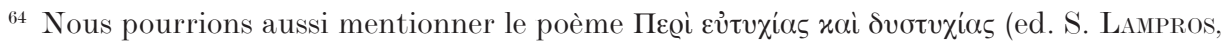

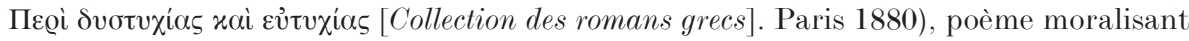
selon H.-G. BEcK, Geschichte der byzantinischen Volksliteratur. Munich 1971, 147. Il s'agit d'un récit à un seul protagoniste où le thème de l'Eros n'apparaît pas. Pourtant, en raison du fait que le récit ne relate qu'une partie seulement de la vie du héros dont nous ignorons le nom et qui reste une figure interchangeable sans aucune attache his- 
Les deux types de récit concernent toujours un récit de vie qui ne se fixe pas dans une réalité historique donnée, mais qui entretient avec elle des liens flottants. Ces liens permettent, occasionnellement, à ce récit de vie de puiser une référence l'accréditant auprès de ses destinataires/récepteurs tout en lui laissant la possibilité de mieux s'adapter à leurs attentes. Pourtant, au-delà de leur degré de fiction ou de stylisation, ces récits se déroulent autour d'un personnage central, dans un récit du deuxième type ou de deux personnages, dans un récit du premier type, d'après le/lesquels le récit est nommé.

Or, afin que cette transposition narrative se matérialise, pour une partie de ces personnages au moins et notamment ceux dotés d'un arrière-plan historique réel, elle a largement recours aux éléments légendaires et mythiques (depuis partie intégrante de leur histoire de par la tradition orale ou écrite de l'imaginaire byzantin), et aux éléments véhiculés par d'autres types de récit comme l'historiographie, les vies des saints ou le roman hellénistique.

Ainsi, à travers ce jeu d'intertextualité ${ }^{65}$, les indications historiques deviennent abstraites, le chrono-tope ${ }^{66}$ se dilate pour pouvoir contenir les

torique spécifique ou générique identifiante, nous considérons qu'il pourrait faire partie des récits de vie partielle, bien que son caractère demeure par excellence didactique.

La Narration de Troie, op.cit, constitue, elle aussi, un cas particulier. Non seulement Pâris tombe amoureux d'une jeune femme dont le nom nous est connu (Hélène), mais en outre la jeune femme remplit un rôle catalyseur pour le déploiement de l'histoire. Ainsi, le thème de l'Eros se voit doté d'une importance capitale pour le déploiement du récit. Parallèlement, à partir du v.763, (quand le couple Pâris- Hélène va quitter le château pour arriver finalement à Troie) la guerre de Troie éclate et avec elle c'est un deuxième héros qui apparaît, le vrai héros homérique de la guerre, Achille. Ce dernier va suppléer la figure de Pâris de sorte que le récit ne s'achève pas avec la mort de Pâris mais sur une lamentation concernant Achille et la gloire éphémère. Pourtant, nous considérons que la Narration de Troie constitue un récit de vie intégrale, vu qu'elle relate le récit de vie de Pâris de sa naissance jusqu'à sa mort, que le couple Pâris-Hélène, certes important, ne constitue qu'une étape de cette vie et qu'Achille incarne la figure du héros adversaire et par conséquent il ne remplit pas le même rôle que remplissait le deuxième protagoniste du récit de vie partielle. De surcroît, Pâris, ne serait-ce que par la tradition homérique, ne constitue pas une figure interchangeable.

${ }_{65}^{6}$ S. MacAlister, Byzantine $12^{\text {th }}$ c. Romances: a Relative Chronology. BMGS 15 (1995) 175-210, ici 181: «it is suggested here, that the romances revival manipulated diverse sources: scientific theory, spirituality, imperial description, tragedy and epic -not to mention but a few- to make their own statements which they directed their contemporary audience's subjective belief system»

${ }^{66}$ M. Bakhtine, Esthétique et théorie du roman (en russe). Moscou 1975, nous utilisons la traduction française par D. Olivier, Paris 1978, ici 237 sq. 
nouveaux éléments introduits par le narrateur ${ }^{67}$ qui, opérant en relation avec son intention significative ${ }^{68}$, voire le sens qu'il veut faire véhiculer par le récit, crée un mythe littéraire qui dépasse amplement le cadre du récit narratif. D'ailleurs, selon R. Barthes, «le mythe ne se définit pas par l'objet de son message, mais par la façon dont il le profère.»

Nous pourrions aussi ajouter que tout héros tend avec le temps à se détacher de la situation, au fur et à mesure qu'il s'installe plus profondément dans la conscience culturelle et se fait valoir comme caractère ou même comme incarnation type d'une idée. Quand on parle de Digénis Akritas, d'Alexandre ou d'Achille il est nécessaire de distinguer et de ne pas confondre la figure du protagoniste d'un récit, le héros littéraire, avec la figure proprement historique qui, peut-être, se trouve à son origine ${ }^{69}$.

En ce qui concerne le «récit de vie intégrale», on aurait pu croire que les choses sont un peu plus compliquées. Le seul choix d'un personnage, d'un nom, d'un fait emprunté au domaine de l'histoire, entraîne automatiquement le créateur à renoncer à l'infini des possibilités qui sont le propre des sujets d'imagination pure, étant donné que leur réalité historique a précédé leur réalité littéraire.

Un double mouvement simultané se déclenche alors, l'un tendant vers sa déshistoricisation et l'autre vers sa mythification, à l'aide d'une suite de signes dilatés, afin que le récit puisse acquérir une nouvelle réalité qui n’est plus historique mais littéraire, formée et puisée en première instance par l'imaginaire, populaire ou littéraire ${ }^{70}$.

${ }^{67}$ Dans ce sens on a pu dire que «la prestation individuelle du poète en face de la force de la tradition apparaît d'abord dans le choix du thème et ensuite dans son traitement particulier par la modification, l'élection et le nouvel agencement des motifs» (E. FrenZEL, Stoff-, Motiv- und Symbolforschung. Berlin 1966, 51).

68 M. Merleau-Ponty, Sur la phénoménologie du langage, in: Eloge de la philosophie. Paris $1963,97$.

${ }^{69}$ Cette distinction déterminante, pourtant, n'a pas été opérante dans le cas de nombreuses études comme celle de H. Grégorre, Michel III et Basile le Macédonien. Byz 5 (1929) 328-340, 329: «L'auteur de Digénis enregistre-t-il les souvenirs du peuple ou bien puiset-il dans des chroniques ses digressions historiques?»; du même, Le tombeau et la date de Digénis Akritas. Byz 6 (1931) 481-508 et Autour de Digénis Akritas. Les cantilènes et la date de la recension d'Andros -Trébizonde. Byz 7 (1932) 287-302, et Notes Complémentaires, ibid., p.317-320;

70 Entre l'imaginaire populaire et la littérature, il y a l'auteur. Etant donné que cet imaginaire se trouve en interaction obligatoire avec la littérature produite par le même contexte social que lui et vice versa, nous ne pouvons concevoir l'imaginaire populaire en tant qu'entité pure, sans aucune influence venant de son contexte socioculturel. Dans cette perspective, la littérature se voit influencée par cet imaginaire, ne serait-ce que parce qu'elle n'en constitue finalement que l'expression stylisée et élaborée de celui-ci. Cette relation interactive est particulièrement visible dans le cas de Digénis Akritas. 
De cette façon pourtant, le récit s'ouvre à cette réalité imaginaire connue par les récepteurs du récit. L'auteur a ainsi face à lui un public prévenu, prêt à la comparaison avec une sorte d'archétype culturel. Cette ouverture s'effectue alors à l'aide des motifs, sorte de toile de fond sur lequel la narration commence à broder son histoire. Le motif, élément non littéraire mais délimitant quelques situations fondamentales, est matière de la littérature. Il désigne une situation de base, impersonnelle, dont les acteurs n'ont pas encore été individualisés. C'est pour cette raison que les motifs sont indépendants du type de récit et qu'ils peuvent s'adapter à l'un ou à l'autre selon des modalités différentes. Ainsi, par exemple, le motif des amours contrariés embrassera à la fois le thème de Leucippé et Clitophon ou celui de Digénis et sa bien aimée: il sera encore une fois une idée générale extra-littéraire mais dont les fixations, de toute évidence, relèvent immédiatement de la littérature. Nous avons affaire à des situations déjà délimitées dans leurs lignes essentielles mais qui restent à l'état de notions générales.

Une étude plus détaillée aurait été fort utile, mais dans le cadre de la présente recherche, nous voudrions nous limiter à l'examen de deux motifs, celui de la jeune femme et celui du combat et de leur développement dans le récit de Digénis Akritas (véhiculé par les versions $\mathbf{G}$ et $\mathbf{E}$ ), d'Alexandre, d'Achille, et de Bélisaire en ne faisant que quelques références aux figures de l'autre type de récit. Le choix de ces deux motifs est dicté tant par le rôle important qu'ils jouent dans le développement du récit que par leur relation intrinsèque.

Le motif de la jeune femme, l'amour du héros pour la jeune femme, est perçu soit comme la raison de ses péripéties (c'est le cas des récits de vie partielle) soit comme l'un de ses exploits afin de se confirmer comme héros (dans le cas des récits de vie intégrale). Ainsi, soit la jeune femme déclenche le récit des péripéties qui arrivent au héros, soit elle fait partie constituante des péripéties qui lui arrivent. Le motif de combat, proliférant dans ces récits, pourrait être divisé en une suite de motifs secondaires plus spécifiques comme le «combat contre des monstres», «combat contre l'Eros», «combat contre le destin» ou le «combat contre des ennemis». Dans tous les cas, pourtant, son rôle est essentiel et par excellence «adjuvant» pour la réalisation de l'image héroïque du protagoniste. Image qui diverge toutefois énormément selon le héros et selon le récit, voire plus précisément selon le but recherché par le récit.

Pour Digénis Akritas, le motif de la jeune femme est d'une importance particulière et constitue l'une des épreuves d'initiation qu'il doit mener à terme, afin de se confirmer comme héros: il doit enlever sa femme et par la 
suite la protéger contre d'éventuels adversaires, sujet qui sera développé par le motif du combat; une fois les adversaires éliminés, la suite du récit nous informe en résumé qu'il est tellement brave qu'il a conquis toute l'Asie Mineure et, avant de nous décrire sa mort soudaine, nous fait la description de la demeure qu'il a construite au bord de l'Euphrate.

Le motif du combat est omniprésent dans le récit, et le conditionne du début à la fin. Digénis Akritas est certes beau, vaillant, brave et sort toujours vainqueur de ses combats. Ainsi il affronte des êtres soit naturels, les Apelates, soit surnaturels, le dragon, soit contre- naturels, Maximô. Pourtant, ce n'est pas lui qui attaque. Son rôle est limité à défendre sa bienaimée ou sa propre vie. Ce sont des combats que mène une figure solitaire (il le dit explicitement: je suis seul et seul j'avance ${ }^{71}$ ) contre des adversaires qui l'empêchent d'exister paisiblement et qui menacent sa vie de couple.

Pour Alexandre, le motif de la jeune femme tient une place secondaire dans le récit. La rencontre avec Roxane est une conséquence de la défaite de Darius ${ }^{72}$ et la fille constitue en quelque sorte son butin ${ }^{73}$ de guerre, étant donné que c'est le père qui la lui donne juste avant de mourir sur le champ de bataille. Il s'agit d'une rencontre arrangée et préparée par une suite de lettres qu'il échange avec la mère de Roxane ${ }^{74}$ et Roxane elle-même ${ }^{75}$. La suite du récit nous raconte leur mariage ${ }^{76}$ et les lettres qu'Alexandre a envoyées à sa mère ${ }^{77}$. Ainsi, dans ce récit le «motif de la jeune femme» trouve un développement différent de celui de Digénis Akritas, parce que son rôle pour la réalisation du destin du héros est différent. Alexandre n'est pas un héros à l'état brut qui cherche à se confirmer à travers sa force et ses exploits. L'histoire l'oblige à conquérir le monde. Alexandre est lui aussi vaillant, mais il se bat suivi de toute une armée et fait parfois appel à la ruse pour vaincre ses adversaires, comme c'est le cas lors de l'épisode de Candaule $^{78}$; parfois, il lui arrive d'avoir peur et même de reconnaître sa défaite, comme c'est le cas dans l'épisode avec la reine Candace. Il reste toujours pourtant ce héros au destin unique qui lui a valu son «affabulation", à savoir le conquérant de l'univers.

${ }^{71} \mathrm{E}, 793,1299$ (Alexiou).

72 v. 4050 (WAGNER).

${ }^{73}$ Il serait intéressant, de noter la similitude de l'emploi de ce motif dans Alexandre avec le motif de Maximô dans Digénis Akritas, où l'amazone offre sa nature féminine au vainqueur Digénis Akritas après la défaite de sa nature masculine.

74 v. 4035 (WAGNER).

75 V. 4104 (WAGNER).

76 v. 4129 (WAGNER).

77 v. 4134 (WAGNER).

78 v. 5139 sq. (WAGNer). 
Dans le récit d'Achille, le motif de la jeune femme prend des dimensions extraordinaires, vu qu'il occupe la plus grande partie du récit ${ }^{79}$ au détriment des autres motifs comme par exemple la «naissance du héros» et sa «croissance miraculeuse» ou ses «combats contre les ennemis». De cette manière, la structure du récit diffère sensiblement de celle de Digénis Akritas ou d'Alexandre, et nous pourrions dire que ce motif constitue son principal exploit. La rencontre est longuement développée et résonne d'un esprit plus romantique, au sens moderne du terme. Ils échangent des lettres, Achille met à l'épreuve l'amour de la femme avec son déguisement, et est finalement aidé lors de l'enlèvement par ses amis ${ }^{80}$. Après, c'est le récit du mariage qui suit ${ }^{81}$ et le tournoi avec le chevalier franc ${ }^{82}$. Il est intéressant que, le chevalier étant perçu comme rival, Achille est décrit par toutes les versions ${ }^{83}$ dans une fureur quasi homérique, fait qui concorde avec notre interprétation. Achille est, lui aussi, brave et courageux mais, comme nous l'avons indiqué, son véritable adversaire n'est que l'Eros. Son passé historique et homérique, passé lointain, de surcroît ignoré par les versions L et $\mathrm{O}$, ne se trouve que dans la fin de la version $\mathrm{N}^{84}$. En revanche, les indications historiques que nous trouvons dans le récit se réfèrent plutôt à la réalité byzantine: les armes, les vêtements, etc.

Dans le récit de Bélisaire, le motif de la jeune femme est absent. Mais il s'agit d'une absence significative, qui marque bien un acheminement différent vers la confirmation du héros. Bélisaire va se confirmer comme un héros qui sauvegarde tout l'Empire contre ses adversaires qui, pourtant, ne sont ni les Anglais ni les Perses ni les Sarrasins. Afin d'être confirmé comme héros, il lui faut combattre la félonie et la ruse qui constituent la malédiction des Romains, comme d'ailleurs nous en informe l'auteur dès le préambule! Dans cette perspective, alors, ses histoires d'amour n'intéressent que peu les récepteurs d'un récit qui se veut didactique. Bélisaire est brave et honnête, mais surtout un très bon et loyal stratège qui connaît bien la tactique militaire. Son destin littéraire et mythique le doit précisé-

\footnotetext{
$79 \mathrm{~N} 651-1565$ (SмIтн).

80 N. 651-1502 (SмIтн).

81 N.1502-1541 (Sмптн).

${ }^{82}$ N.1542-1596 (Sмiтн).

83 N.1572 (Smith), O.662 (Sмith), L.1170 (Hesseling)

84 N.1860-1916 (Sмiтh). Certes, dès le VI e s. déjà, avec Malalas (ed. I. Thurn, Ioannis Malalae Chronographia [CFHB 35]. Berlin-New York 2000, ch. 28, p. 99), le motif de l'amour de la jeune fille qui conduit le héros à la mort est connu, mais il est présenté d'une manière très différente. Nous trouvons la même version dans l'histoire de Constantin Manassès (ed. O. Lampsidis, Constantini Manassis Breviarium [CFHB 36.1/2]. Athènes 1996, v. 1381-1409).
} 
ment à son efficacité stratégique qui a su venir à bout des Vandales, des Perses et des Goths et a pu lui permettre de défendre l'Empire tout en restant loyal envers son empereur. Ainsi, sa mythification a commencé dès son époque, déjà, par les historiens Procope de Césarée ${ }^{85}$ et Agathias Scholastique. Par la suite, l'imaginaire lui a attribué des exploits qui ne peuvent chronologiquement être les siens, mais qui, par leur force mythifiante, le projetaient comme héros honnête et vaillant, capable de répondre aux attentes de la société.

Par l'examen, certes non exhaustif, de ces exemples, nous avons d'abord essayé de comprendre, étant donné les limites de l'application du terme de roman dans la production littéraire de Byzance, si, à l'époque, il existait une catégorie analogue à celle du roman tel que nous le concevons actuellement, et si oui, laquelle et quels ont été les textes qu'elle recouvrait. Ainsi, nous avons pu constater que des textes qui n'entrent pas facilement dans la catégorie du roman, au moins tel que celui-ci est appréhendé par la littérature contemporaine, partagent entre eux, mais également avec les textes classés sans difficulté sous la dénomination «roman», deux éléments constituants: la narration d'un récit de vie construit, relevant donc de la fiction, et le pacte de l'illusion qui régit cet acte de raconter. Il s'agit d'un récit qui, même si parfois il puise son inspiration dans des faits historiques, ne fait pas appel à l'image du réel et à la vraisemblance mais plutôt à «l'effet de réel» derrière lequel se dissimile la convention première du récit: la fiction. Plus précisément, le but de la narration n'est ni de décrire la réalité ni de faire un rapport sur des faits historiques mais plutôt de construire et de porter le monde supposé et proposé par la fiction du récit en adoptant un discours réaliste.

Par la suite, nous avons tenté de cerner les caractéristiques des différents textes que nous trouvons dans cette catégorie. Subséquemment, nous avons essayé de comprendre quelle est la différence constituante du texte qui rend distincte une figure héroïque - par exemple celle de Digénis Akritas - des autres figures littéraires, Alexandre ou Achille, Chariclès ou Callimaque. Cela nous a conduits dans un premier temps à faire émerger deux mouvements opposés, l'un allant de l'histoire au mythe par un effet, pour ainsi dire, de déshistorification -pour des héros tels Alexandre et Bélisaire-,

85 Concernant Procope, nous pouvons constater un double mouvement de mythification de Bélisaire, un positif dans les Guerres et un négatif dans Anecdotes. Sur la figure de Bélisaire, victime de l'envie, voir dans l'histoire de Constantin Manassès (v. 3180-3210 LAMPSIDIS). 
et l'autre du mythe à l'histoire, par un effet d'historification -Digénis Akritas ou Achille. Notre étude a aussi permis de mettre en évidence pour les figures telles que Hysminias ou Dosiclès l'impossibilité de la métamorphose de leurs destins en mythe autre que littéraire, en raison même de leur littéralité, de leur fixité, de leurs liens étroits avec le contexte dans lequel ils se développent et qui les empêchent de s'ériger en figures mythiques dépassant leur cadre littéraire. Avec cette perspective, nous avons ensuite démontré l'existence de deux sous-catégories: a. le récit de vie partielle, récit à «thème de situation», à deux protagonistes, où le motif de «l'amour pour la jeune femme» constitue le motif prédominant, et dont les héros restent des figures interchangeables précisément en raison de leur dépendance directe à l'égard du contexte littéraire dans lequel ils évoluent; $\mathbf{b}$. le récit de vie intégrale, récit à «thème de héros», à un seul protagoniste, qui entretient des liens, bien que lâches, avec un certain fond de réalité historique et dont la figure principale est dotée de toutes les particularités complexes d'une figure suffisamment individualisée.

A la différence des récits historiques, biographiques ou même hagiographiques qui eux aussi constituent des récits de vie, les récits de vie partielle ou intégrale appartenant à la catégorie de la narration sont déterminés par la convention première qui est celle de la fiction et qui décide l'agencement interne du récit.

Or, les figures des héros des récits de vie partielle, issues de l'imaginaire de l'auteur dont elles dépendent directement, se voient dépouillées de leurs traits individualisants et restent intimement liées et ancrées à la littéralité dans laquelle cet imaginaire les a fait évoluer. Les figures des héros des récits de vie intégrale sont également soumises à la même convention de la fiction et au traitement littéraire que l'auteur choisit. Cependant, en raison du fait qu'elles constituent des figures principales héritées de l'histoire, du mythe ou de la tradition, les figures héroïques des récits de vie intégrale conservent leurs traits individuels et par conséquent ne sont que partiellement tributaires de cet imaginaire.

Enfin, les divers éléments mis au jour au cours de l'examen de ces exemples amènent à conclure qu'au-delà de leur parenté structurelle, chacun de ces récits obéit à sa propre logique et, par extension, développe, de sa propre manière, les motifs qui sont communs à toutes les narrations, mais à des degrés divers selon le type et le sens du récit. 
